\title{
Index Theorem and Random Matrix Theory for Improved Staggered Quarks
}

\author{
Eduardo Follana* \\ University of Glasgow \\ E-mail: e.follana@physics.gla.ac.uk
}

\author{
Alistair Hart \\ University of Edinburgh \\ E-mail: a.hart@ed.ac.uk
}

\section{Christine T.H. Davies}

University of Glasgow

E-mail: c.davies@physics.gla.ac.uk

\begin{abstract}
We study various improved staggered quark Dirac operators on quenched gluon backgrounds in lattice QCD. We find a clear separation of the spectrum of eigenvalues into high chirality, wouldbe zero modes and others, in accordance with the Index Theorem. We find the expected clustering of the non-zero modes into quartets as we approach the continuum limit. The predictions of random matrix theory for the epsilon regime are well reproduced. We conclude that improved staggered quarks near the continuum limit respond correctly to QCD topology.
\end{abstract}

XXIIIrd International Symposium on Lattice Field Theory

25-30 July 2005

Trinity College, Dublin, Ireland

\footnotetext{
*Speaker.
} 


\section{Motivation}

The low energy regime of Quantum Chromodynamics (QCD) exhibits a rich and interesting phenomenology, including the $U_{A}(1)$ axial anomaly, chiral symmetry breaking and the topological properties of the theory. There are a number of detailed predictions of the properties of these lowlying modes, such as the existence of an Index Theorem [1,2] and the distribution of the first few eigenvalues in fixed topological charge sectors $[3,4,5,6,7,8]$.

Any correct discretization of QCD must agree with those predictions close enough to the continuum limit. Here we show that this is already the case for improved staggered quarks at the lattice spacings typically used in present-day simulations. In particular, we show that improved staggered fermions do respond correctly to the gluonic topological charge, and discuss why some confusion on this issue exists in the literature. Here we expand the work in $[9,10]$. For more detailed results see [11]. Related work has been presented in $[12,13]$.

\section{Improved Staggered Dirac Operators}

The massless, gauge-invariant, ONE-LINK staggered Dirac operator on a $d=4$ dimensional Euclidean lattice with spacing $a$ is

$$
\mathbb{D}(x, y)=\frac{1}{2 a u_{0}} \sum_{\mu=1}^{d} \eta_{\mu}(x)\left[U_{\mu}(x) \delta_{x+\hat{\mu}, y}-H . c .\right], \quad \eta_{v}(x)=(-1)^{\sum_{\mu<v} x_{\mu}}
$$

with $u_{0}$ an optional tadpole-improvement factor given, in our case, by the fourth root of the mean plaquette. ID is antihermitian and obeys a remnant of the continuum $\gamma_{5}$ anticommutation relation, $\{\not D, \varepsilon\}=0$, with $\varepsilon(x)=(-1)^{\sum_{\mu=1}^{d} x_{\mu}}$. As in the continuum case, its spectrum is therefore purely imaginary, with eigenvalues occurring in complex conjugate pairs, $\left\{ \pm i \lambda_{s}, \lambda_{s} \in \mathbb{R}\right\}$. The corresponding action describes $N_{t}=4$ "tastes" of fermions which interact via unphysical "taste breaking" interactions that vanish in the continuum limit as $a^{2}$. In such limit there is an $S U\left(N_{t}\right) \otimes S U\left(N_{t}\right)$ chiral symmetry, and the spectrum is therefore $N_{t}$-fold degenerate. There is also an exact Index Theorem. At finite lattice spacing the chiral symmetry group is reduced to $U(1) \otimes U(1)$ and we do not expect to see this picture, for example there will not be an exact Index Theorem anymore.

In addition to the ONE-LINK operator, we have also studied several improved staggered operators, the so called ASQTAD, FAT7XASQ and HYP , designed to suppress the taste-changing interactions $[14,15,16]$.

\section{Details of the Simulation}

We use a quenched, $S U(3)$ gluonic action that is both tree level Symanzik and tadpole improved [17, 18, 19, 20, 21]. It differs from the one used by the MILC Collaboration [22] only in small one loop radiative corrections. We have generated several ensembles of around 1000 configurations.

We measure the gluonic topological charge $Q$ using two different cooling methods, to look for consistency. We discard the configurations for which both methods disagree. In the $a=0.093 \mathrm{fm}$ ensemble fewer than $10 \%$ of the configurations have an ambiguous topological charge. For the 
Improved Glue $\left(a \approx 0.077 \mathrm{fm}, \mathrm{V}=20^{4}\right)$
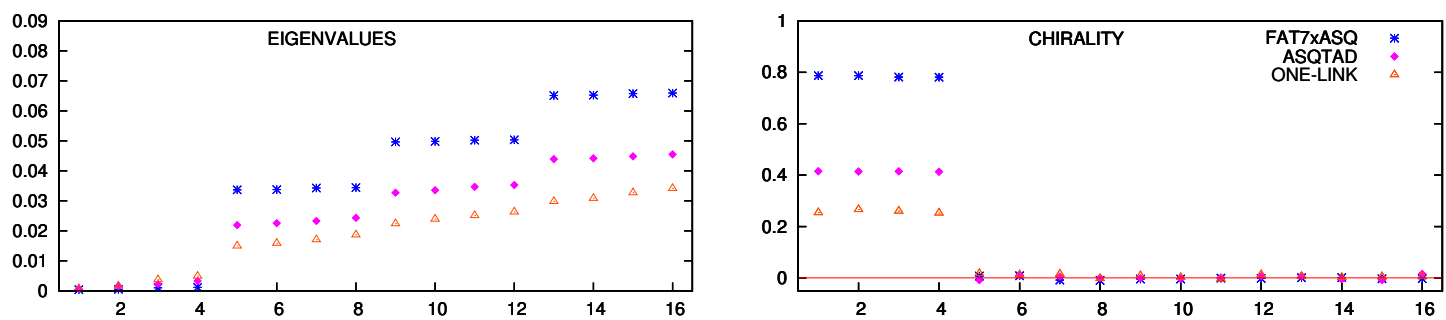

Figure 1: The positive half of a typical eigenmode spectrum for a configuration of $Q=2$, in lattice units. The $x$-axis is eigenvalue number. The HYP operator gives results very similar to FAT7XASQ, and they are not shown for clarity.

finest ensemble, $a=0.077 \mathrm{fm}$, this number goes down to around $2 \%$. We stress that we only use cooling to determine the topological charge. All the Dirac spectrum measurements are done on the original thermalized ("hot") configurations.

\section{Index Theorem}

We begin our analysis by qualitatively comparing the low-lying modes of various staggered quark operators on a typical gauge background selected from those with $Q=2$. In Fig. 1 we show both the eigenvalue and the chirality of the first sixteen (positive) eigenmodes. Near the continuum limit we expect to see first $2|Q|=4$ near-zero modes with their chirality renormalised slightly away from unity. The remaining modes should have chirality near zero and divide into almost degenerate quartets. The spectrum looks quite continuum-like for all Dirac operators and we see a clear Index Theorem. For the improved operators we also see a very clear quadruple degeneracy in the nonzero modes. The renormalisation of the chirality away from 1 is small for the improved Dirac operators (around $Z=1.2$ ), becoming as large as $Z \approx 4$ for the ONE-LINK fermions. This result is fairly typical of operator renormalisation for staggered quarks [23, 24, 25].

\section{Comparison with Random Matrix Theory}

The chiral symmetries of staggered quarks are more complicated than for continuum QCD. At finite lattice spacing there are $N_{t}^{2}=16$ pion states, and only one of these becomes massless in the chiral limit, the "Goldstone pion", with mass $M_{G}$. The 15 remaining states have masses $\sim M_{N G}$ arising from the taste breaking interactions. The (chirally extrapolated) masses are $\mathscr{O}\left(a^{2}\right)$ and therefore zero only in the continuum limit.

There are potentially two universal regions for different parameters of the system:

$$
\begin{aligned}
\varepsilon \text {-regime: } & \left(\Lambda_{Q C D}\right)^{-1} \ll L \ll\left(M_{N G}\right)^{-1}, \\
\mathcal{\varepsilon}^{\prime} \text {-regime: } & \left(M_{N G}\right)^{-1} \ll L \ll\left(M_{G}\right)^{-1} .
\end{aligned}
$$

In the first one the chiral symmetries are as in the continuum, corresponding to an effective theory of 16 massless pions. The low-lying non-zero modes have a near $N_{t}$-fold degeneracy, and follow the distributions corresponding to continuum QCD (and chiral lattice fermions [26, 27, 28, 29, 30, 31]). 
At finite lattice spacing there is a second, $\varepsilon^{\prime}$-regime, corresponding to an effective theory with a single massless pion. There is not even approximate restoration of the continuum symmetries, and the associated RMT has only $U(1) \otimes U(1)$ chiral symmetry. The universal predictions for the eigenvalues are therefore strikingly different from those for continuum QCD. In particular, the predictions are the same for all sectors of topological charge [32]. Presumably it is this regime that was studied in [33, 34, 35, 36, 37]. With the coarse lattices and unimproved gauge ensembles used in these studies, they observed no sensitivity of the eigenvalue spectra to $Q$, leading to the incorrect folklore that staggered quarks are "blind to the topology". To see the continuum chiral symmetries, there must be a sufficiently large mass gap between the heaviest pion and the lightest of the other hadrons. This requires the use of improved gauge and fermion actions and a sufficiently small lattice spacing. At coarse lattice spacings, with unimproved fermions, $M_{N G} \approx \Lambda_{Q C D}$ and there will be no $\varepsilon$-regime.

We separately fit the individual cumulative spectral densities to the predictions from RMT. These one parameter fits yield a prediction for the chiral condensate. We show some examples of the fits in Figs. 2. It is clear that the improved Dirac operators match the predictions of RMT very closely over the full range of lattice spacings. By contrast, even at $a=0.077 \mathrm{fm}$ the ONE-LINK Dirac operator still shows significant discrepancies. The fits also show clearly the expected variation with topological charge. Each fit gives a value for the bare chiral condensate, and we find all of them to be broadly consistent with each other.

\section{Conclusions and Outlook}

We have studied the low-lying eigenmodes for the staggered lattice Dirac operator, using a range of improved and unimproved versions. The improved operators pass all the tests. In particular, and contrary to previous accepted wisdom, such fermions do respond exactly as expected to the gluonic topological charge, and in a way identical to continuum QCD and other lattice formulations of the Dirac operator.

We have seen that for improved operators the eigenvalue spectrum divides cleanly into nearzero and non-zero modes. The near-zero modes are characterised by a uniformly high chirality, with their relative number fixed by the Atiyah-Singer Index Theorem. The non-zero modes, by contrast, have chirality that is near zero and they divide into near degenerate quartets. In addition, the low-lying non-zero modes follow closely the universal distributions predicted by random matrix theory.

\section{Acknowledgments}

This research is part of the EU integrated infrastructure initiative hadron physics project under contract number RII3-CT-2004-506078.

We thank: Ph. de Forcrand for his topological charge measurement code; A. Hasenfratz for help in implementing the HYP operator; G.P. Lepage, P. Damgaard and G. Akemann for useful discussions. E.F., C.T.H.D. and Q.M. are supported by PPARC and A.H. by the U.K. Royal Society. The eigenvalue calculations were carried out on computer clusters at Scotgrid and the Dallas Southern Methodist University. We thank David Martin and Kent Hornbostel for assistance. 

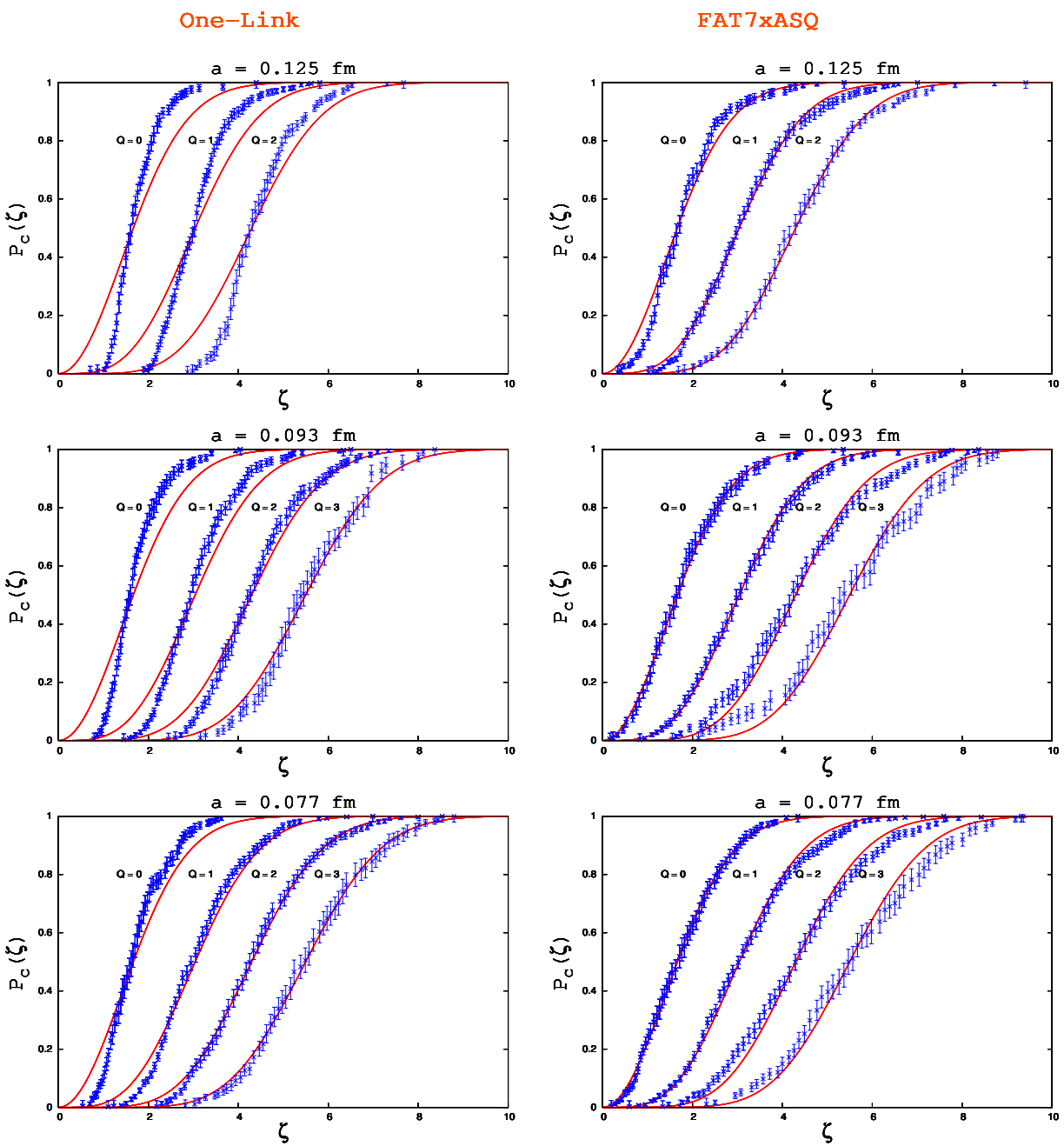

Figure 2: Comparison of the unfolded eigenvalue distribution with RMT for the lowest eigenvalue for different topological charges. The lattice size is kept at $a L \approx 1.5 \mathrm{fm}$

\section{References}

[1] M. Atiyah and I. Singer, Bull. Amer. Math. Soc. 69, 422 (1963).

[2] M. Atiyah and I. Singer, Ann. Math. 87, 596 (1968).

[3] H. Leutwyler and A. Smilga, Phys. Rev. D46, 5607 (1992).

[4] E. V. Shuryak and J. J. M. Verbaarschot, Nucl. Phys. A560, 306 (1993), [hep-th/9212088].

[5] P. H. Damgaard, Nucl. Phys. Proc. Suppl. 106, 29 (2002), [hep-lat/0110192].

[6] G. Akemann and P. H. Damgaard, Phys. Lett. B583, 199 (2004), [hep-th/0311171].

[7] S. M. Nishigaki, P. H. Damgaard and T. Wettig, Phys. Rev. D58, 087704 (1998), [hep-th/9803007].

[8] P. H. Damgaard and S. M. Nishigaki, Phys. Rev. D63, 045012 (2001), [hep-th/0006111], see corrections at hep-th/0006111v2.

[9] E. Follana, A. Hart and C. T. H. Davies, Phys. Rev. Lett. 93, 241601 (2004), [hep-lat/0406010]. 
[10] E. Follana, Nucl. Phys. Proc. Suppl. 140, 141 (2005), [hep-lat/0409062].

[11] E. Follana, A. Hart, C. T. H. Davies and Q. Mason, Phys. Rev. D 72, 054501 (2005), [hep-lat/0507011].

[12] K. Y. Wong and R. M. Woloshyn, hep-lat/0407003.

[13] K. Y. Wong and R. M. Woloshyn, Phys. Rev. D71 (2005), [hep-lat/0412001].

[14] S. Naik, Nucl. Phys. B316, 238 (1989).

[15] G. P. Lepage, Phys. Rev. D59, 074502 (1999), [hep-lat/9809157].

[16] F. Knechtli and A. Hasenfratz, Phys. Rev. D63, 114502 (2001), [hep-lat/0012022].

[17] G. Curci, P. Menotti and G. Paffuti, Phys.Lett. B130, 205 (1983).

[18] Erratum:, G. Curci, P. Menotti and G. Paffuti, Phys.Lett. B135, 516 (1984).

[19] M. Luscher and P. Weisz, Commun. Math. Phys. 97, 59 (1985).

[20] Erratum:, M. Luscher and P. Weisz, Commun. Math. Phys. 98, 433 (1985).

[21] M. Alford, W. Dimm, G. Lepage, G. Hockney and P. Mackenzie, Phys. Lett. B361, 87 (1995), [hep-lat/9507010].

[22] HPQCD, C. T. H. Davies et al., Phys. Rev. Lett. 92, 022001 (2004), [hep-lat/0304004].

[23] J. Hein, Q. Mason, G. Lepage and H. Trottier, Nucl. Phys. Proc. Suppl. 106 (2002), [hep-lat/0110045].

[24] H. Trottier, G. Lepage, P. Mackenzie, Q. Mason and M. Nobes, Nucl. Phys. Proc. Suppl. 106 (2002), [hep-lat/0110147].

[25] W. Lee and S. Sharpe, Phys. Rev. D66, 114501 (2002), [hep-lat/0208018].

[26] R. G. Edwards, U. M. Heller, J. E. Kiskis and R. Narayanan, Phys. Rev. Lett. 82, 4188 (1999), [hep-th/9902117].

[27] R. G. Edwards, U. M. Heller, J. E. Kiskis and R. Narayanan, Phys. Rev. D61, 074504 (2000), [hep-lat/9910041].

[28] P. H. Damgaard, R. G. Edwards, U. M. Heller and R. Narayanan, Phys. Rev. D61, 094503 (2000), [hep-lat/9907016].

[29] P. Hasenfratz, S. Hauswirth, T. Jorg, F. Niedermayer and K. Holland, Nucl. Phys. B643, 280 (2002), [hep-lat/0205010].

[30] W. Bietenholz, K. Jansen and S. Shcheredin, JHEP 07, 033 (2003), [hep-lat/0306022].

[31] L. Giusti, M. Luscher, P. Weisz and H. Wittig, JHEP 11, 023 (2003), [hep-lat/0309189].

[32] P. H. Damgaard, private communication, 2004.

[33] M. E. Berbenni-Bitsch, S. Meyer, A. Schafer, J. J. M. Verbaarschot and T. Wettig, Phys. Rev. Lett. 80, 1146 (1998), [hep-lat/9704018].

[34] P. H. Damgaard, U. M. Heller and A. Krasnitz, Phys. Lett. B445, 366 (1999), [hep-lat/9810060].

[35] M. Gockeler, H. Hehl, P. E. L. Rakow, A. Schafer and T. Wettig, Phys. Rev. D59, 094503 (1999), [hep-lat/9811018].

[36] P. H. Damgaard, U. M. Heller, R. Niclasen and K. Rummukainen, Phys. Rev. D61, 014501 (2000), [hep-lat/9907019].

[37] P. H. Damgaard, U. M. Heller, R. Niclasen and K. Rummukainen, Phys. Lett. B495, 263 (2000), [hep-lat/0007041]. 